\title{
Nanostructured Diamond-Like Carbon Films Grown by Off-Axis Pulsed Laser Deposition
}

\author{
Seong Shan Yap, ${ }^{1}$ Chen Hon Nee, ${ }^{1}$ Seong Ling Yap, ${ }^{2}$ and Teck Yong Tou ${ }^{1}$ \\ ${ }^{1}$ Faculty of Engineering, Multimedia University, 63100 Cyberjaya, Selangor, Malaysia \\ ${ }^{2}$ Department of Physics, Faculty of Science, University of Malaya, 50603 Kuala Lumpur, Malaysia \\ Correspondence should be addressed to Seong Shan Yap; seongshan@gmail.com
}

Received 4 September 2015; Accepted 28 October 2015

Academic Editor: Weidong Zhou

Copyright (c) 2015 Seong Shan Yap et al. This is an open access article distributed under the Creative Commons Attribution License, which permits unrestricted use, distribution, and reproduction in any medium, provided the original work is properly cited.

\begin{abstract}
Nanostructured diamond-like carbon (DLC) films instead of the ultrasmooth film were obtained by pulsed laser ablation of pyrolytic graphite. Deposition was performed at room temperature in vacuum with substrates placed at off-axis position. The configuration utilized high density plasma plume arriving at low effective angle for the formation of nanostructured DLC. Nanostructures with maximum size of $50 \mathrm{~nm}$ were deposited as compared to the ultrasmooth DLC films obtained in a conventional deposition. The Raman spectra of the films confirmed that the films were diamond-like/amorphous in nature. Although grown at an angle, ion energy of $>35 \mathrm{eV}$ was obtained at the off-axis position. This was proposed to be responsible for subplantation growth of $\mathrm{sp}^{3}$ hybridized carbon. The condensation of energetic clusters and oblique angle deposition correspondingly gave rise to the formation of nanostructured DLC in this study.
\end{abstract}

\section{Introduction}

Carbon-based nanomaterials such as C60, carbon nanotubes, and graphene have fascinating structure based on $\mathrm{sp}^{2}$ hybridization. $\mathrm{sp}^{3}$ phase containing diamond or diamondlike structure requires high temperature high pressure condition or metastable states for formation and is thus more difficult to be incorporated in devices. Pulsed laser deposition (PLD) has been used in growing hydrogen-free amorphous carbon (a-C) or diamond-like carbon (DLC) films which has a substantial amount of $\mathrm{sp}^{3}$ hybridization at room temperature in vacuum owing to the highly energetic plasma plume $[1,2]$. The carbon species produced by a nanosecond laser reached up to few hundred $\mathrm{eV}$ and thus DLC films growth can be explained by subplantation growth by hyperthermal species [3]. In this mechanism, C species with high energy are needed to undergo a shallow subsurface implantation that enables $\mathrm{sp}^{3}$ bonds formation and densification. The resultant DLC films are smooth and have very low coefficient of friction $[4,5]$. Ultrasmooth DLC films are excellent tribological coating. On the other hand, nanostructured carbon films are normally grown at high temperature [6-8] or in background gas in PLD [7, 9-14]. Nanostructured carbon films are also grown by using chemical vapor deposition [7] and ion-assisted process [8] at high temperature. However, the nanostructured films normally contain only a small fraction of $\mathrm{sp}^{3}$ hybridized carbon network and thus are more graphitic. Nanostructured DLC films may warrant some new applications in sensing or energy storage where large surface area is needed.

In this work, nanostructured DLC instead of smooth films are produced in off-axis PLD at room temperature. The properties of DLC nanostructured film are compared with ultrasmooth DLC films and the configuration that enables the formation is discussed. The growth configuration can be extended to induce nanostructured growth for other materials.

\section{Experimental}

Pulsed laser ablation of pyrolytic graphite (99.999\% purity, Kurt J. Lesker) was performed by using Nd:YAG laser (EKSPLA, $355 \mathrm{~nm}$ ) in background pressure of $10^{-6}$ Torr. Deposition was performed with laser fluence of $12.5 \mathrm{~J} / \mathrm{cm}^{2}$ at room temperature. Laser beam was directed at an incident 


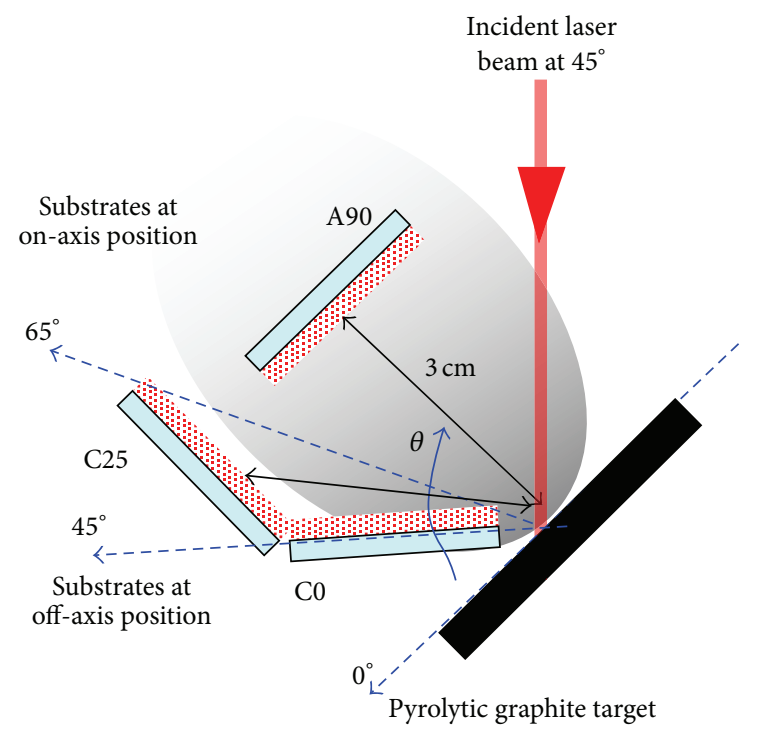

Figure 1: On-axis (A90) and off-axis (C0 and C25) deposition configuration for diamond-like carbon. Plasma plume was collected at different angle, $\theta$ from the target.

angle of $45^{\circ}$ to the target. Precleaned Si (100) substrate was placed at three positions in separate experiments as shown in Figure 1. In the first deposition, a substrate was placed at onaxis position, parallel and at $3 \mathrm{~cm}$ from the target; the sample is denoted as sample A90. $\theta$ is the angle between the target surface and the laser-produce plasma plume. In subsequent depositions, Si substrate was placed at two different offaxis positions (Figure 1): Si substrate was placed at $\theta=45^{\circ}$ and $5 \mathrm{~mm}$ from the ablation area (sample $\mathrm{C} 0$ ), while, in another deposition, Si substrate was placed perpendicular to the target but centered at $3 \mathrm{~cm}$ from the target (sample C25). Deposition was performed at $10 \mathrm{~Hz}$ repetition rate for 6000 pulses for all the experiments. The effective angle where the plasma plume/species arrived onto the substrate is defined as $\alpha$ with reference to the substrate. So $\alpha=0^{\circ}$ for sample C0 where plasma plume is parallel to the substrate, $\alpha=45^{\circ}$ to $25^{\circ}$ for sample $\mathrm{C} 25$, and $\alpha=90^{\circ}$ for sample A90.

An ion probe was used to measure the velocity and kinetic energy of carbon species ejected at different angle $(\theta)$ from the target at fixed laser fluence. The as-deposited samples were analyzed by using profilometer (Mahr), microRaman spectroscopy (514 nm, Renishaw-MicroRaman 2000), and atomic force microscope (AFM) (Nanoscope III, Digital Instruments).

\section{Results and Discussions}

3.1. Ion Energy. The time of flight of the ions arriving at different plume angle $\theta$ was captured by the ion probe at $3 \mathrm{~cm}$ from the target (Figure 2) and the ion signals collected at different angles of the plume are shown in Figure 3. The angular dependence of peak velocity and kinetic energy of the ions are shown in Table 1 . The ion energy and the intensity of the signal reduced when the plume angle decreased. The
TABLE 1: The velocity and kinetic energy of the ions collected at different angle.

\begin{tabular}{lcc}
\hline Angle (degree) & Velocity $\left(\mathrm{km} \mathrm{s}^{-1}\right)$ & Kinetic energy $(\mathrm{eV})$ \\
\hline 30 & 22.71 & 32.14 \\
45 & 23.73 & 35.10 \\
60 & 33.48 & 69.86 \\
90 & 46.88 & 136.92 \\
\hline
\end{tabular}

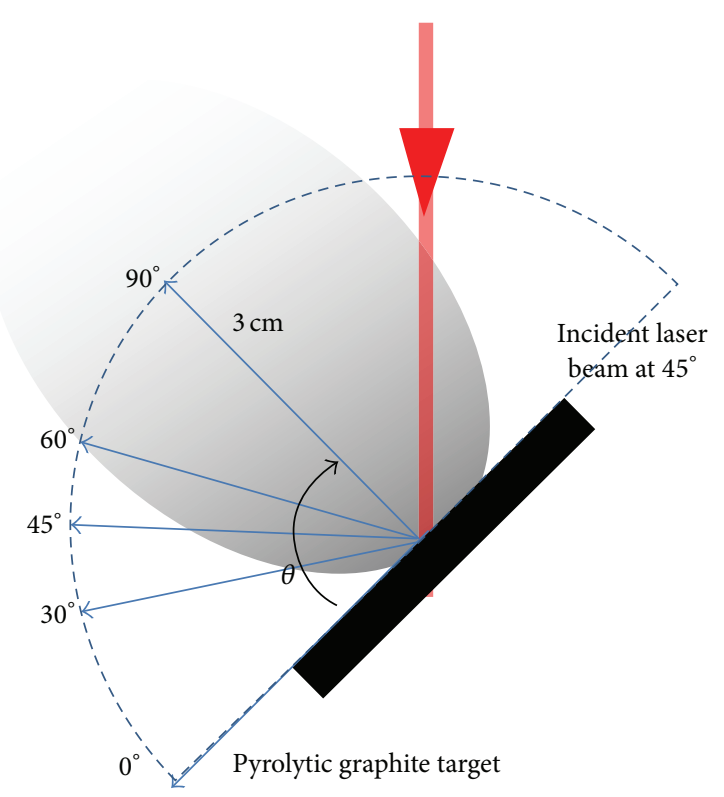

FIGURE 2: Ion probe position for ions detection in vacuum chamber.

results indicate that ion density and ion energy were lower at smaller plume angle.

In the typical on-axis position (A90), where the substrate was parallel to the target, plasma plume with high energy would propagate perpendicular to the target surface towards substrate A90 as in any conventional pulsed laser deposition process. The ion density and energy at this angle $\left(90^{\circ}\right)$ were measured to be highest. For sample $\mathrm{C} 0$, the ion energy was $35 \mathrm{eV}$ and, for C25, the ion energy range was $\sim 35-70 \mathrm{eV}$.

3.2. DLC Films. The morphology of the deposited layers is shown in Figure 4. An ultrasmooth surface with roughness of $0.12 \mathrm{~nm}$ was obtained for the on-axis film A90. On the other hand, small numbers of nanosized particles were detected on smooth film in sample C25 and nanostructured films were obtained in off-axis sample C0. Cross section analysis of the nanostructures gives a lateral size of $\sim 50 \mathrm{~nm}$ and height of $\sim 5 \mathrm{~nm}$. However, it was noted that the adherence of nanostructured film $\mathrm{C} 0$ was poor.

Despite the difference in morphologies, all of the samples displayed the characteristics Raman spectra for amorphous carbon films (Figure 5). At $514 \mathrm{~nm}$ excitation wavelength, the light source coincides with the optical absorption of $\pi \rightarrow$ $\pi^{*}$ for $\mathrm{sp}^{2}$ sites. When these sites are excited, the Raman signal for DLC generally has a broad spectrum, which is asymmetrically centred at $\sim 1560 \mathrm{~cm}^{-1}$ in contrast to sharp 


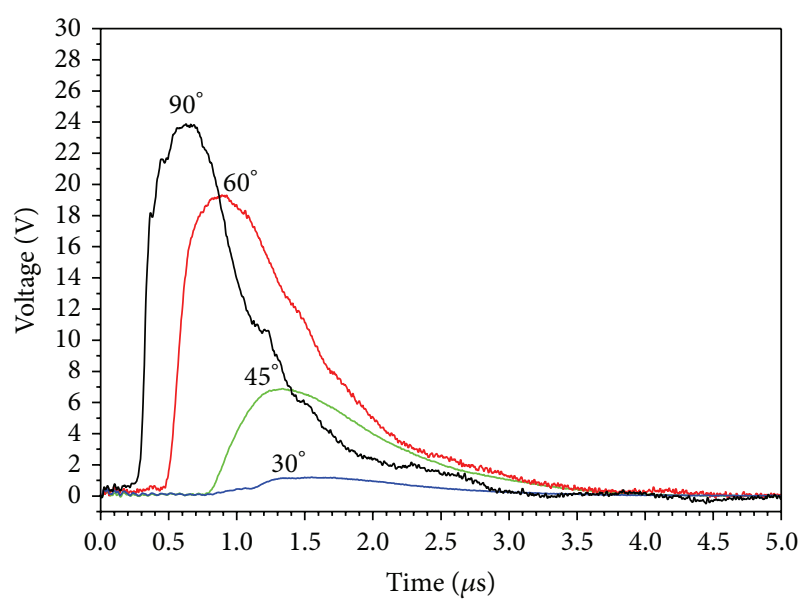

FIgURE 3: Ions signal collected at different plume angle.

peaks of diamond and graphite. The broad Raman spectrum of DLC can be deconvoluted into 2 smooth curves, which are known as the $D$-peak and the $G$-peak [15]. The $D$-peak is the disorder-induced band that arises due to the breathing modes of $\mathrm{sp}^{2}$ in the ring while the $G$-peak is ascribed to the stretching of all pairs of $\mathrm{sp}^{2}$ in the ring and chains. Although the visible Raman spectra depend formally on the configuration of the $\mathrm{sp}^{2}$ sites in $\mathrm{sp}^{2}$-bonded clusters, $\mathrm{sp}^{2}$ clustering is controlled by $\mathrm{sp}^{3}$ fraction in as-deposited amorphous carbon films. Based on the effects, a three-stage model has been reported to account for the amorphization path of graphite in these films. This results in two important parameters: the intensity ratio $I_{D} / I_{G}$ and the $G$-peak position which serve as a useful guide to the amount of $\mathrm{sp}^{3}$ fraction in the films.

In order to compare the samples, the spectra obtained were fitted with 2 Gaussian curves (in red) in Figure 5. The intensity ratio $I_{D} / I_{G}$ and $G$-peak position were extracted and tabulated in Table 2. For the samples grown by PLD, the onaxis film A90 has the lowest $I_{D} / I_{G}$ ratio, followed by $\mathrm{C} 25$ and then nanostructured film $\mathrm{C} 0$. The Raman spectra of a commercially sputtered DLC film were also obtained and the fitting yields $I_{D} / I_{G}$ ratio of 0.73 , higher than the three films grown in this work. It is also noted that sample C25 is thinner than A90 or C0, as seen in the higher intensity peak of the Si substrate at about $960 \mathrm{~cm}^{-1}$. Based on both $I_{D} / I_{G}$ and $G$ peak position, the films grown in this study fell within stage 2 and stage 3 in the amorphization trajectory [15], which corresponds to $\mathrm{sp}^{3}$ fraction in the range of $0-20 \%$ for $\mathrm{C} 0$ and C25 and 20-85\% for sample A0.

Alternatively, the Raman spectrum can also be fitted mathematically by a Breit-Wigner-Fano (BWF) with or without a Lorentzian [16], which is given by

$$
I(\omega)=\frac{I_{0}\left[1+2\left(\omega-\omega_{0}\right) / Q \Gamma\right]^{2}}{1+\left[2\left(\omega-\omega_{0}\right) / \Gamma\right]^{2}}+(a+b \omega),
$$

where $I(\omega)$ is the Raman intensity as a function of Raman shift, $\omega, \omega_{0}, I_{0}$, and $\Gamma$ are the peak position, peak height, and full width at half maximum (FWHM), respectively, and $a$ and
TABLE 2: The characteristics of Raman spectra of DLC films deposited at different positions.

\begin{tabular}{ccccc}
\hline \multicolumn{2}{c}{ Sample } & C0 & C25 & A90 \\
\hline \multirow{2}{*}{ Gaussian fit } & $G$-peak $\left(\mathrm{cm}^{-1}\right)$ & 1558.47 & 1552.99 & 1557.72 \\
& $I_{D} / I_{G}$ & 0.68 & 0.50 & 0.26 \\
\hline \multirow{2}{*}{ BWF fit } & $Q$ & -3.02 & -6.27 & -8.96 \\
& $I_{D} / I_{G}$ & 0.16 & 0.027 & $\sim 0$ \\
\hline \multicolumn{4}{c}{ Increasing $\longrightarrow$} \\
\hline \multicolumn{2}{c}{$\mathrm{sp}^{3}$ fraction } & 11.10 & 9.53 & 6.86 \\
\hline \multicolumn{4}{c}{ Clusters area } & \multicolumn{3}{c}{ Decreasing $\longrightarrow$} \\
\hline
\end{tabular}

$b$ are constant. The factor $Q$ represents the skewness of the spectra, and a negative value serves as an indication of high $\mathrm{sp}^{3}$ film, such as for $\mathrm{A} 0$, and the $\mathrm{sp}^{3}$ fraction reduced for sample C25 and C0. The same trend was observed by using Gaussian fit.

In addition to the fraction of $\mathrm{sp}^{3}$ in the films, $I_{D} / I_{G}$ is also related to the cluster diameter $\left(L_{a}\right)$ in the films. In highly disordered amorphous carbon, $I_{D} / I_{G}=C^{\prime}(\lambda) L_{a}{ }^{2}$, where $C^{\prime}(\lambda)$ is a excitation wavelength-dependence constant [15]. Thus, $C^{\prime}(514 \mathrm{~nm}) \sim 0.0055$ in this study. The deduced cluster sizes are shown in Table 2, where the value increased for the off-axis samples. Thus, the slow species in the plume that travelled at small angle upon ablation results in larger clusters formation.

3.3. Discussions. The morphology or formation of nanostructures has often been related to deposition in the background gas, heated substrate, or deposition of incident carbon species at an angle. In the presence of background gas, plasma plume expansion is confined and collisions among ablated species occur and clusters or nanoparticles formation can be enhanced [9-12]. At the more extreme regime where the ion energy and the confinement of the background are higher, DLC nanoparticles with size of $10 \mathrm{~nm}$ were obtained by using femtosecond laser ablation in liquid [17]. It is interesting to note that the Raman spectra obtained here are similar to those obtained from the DLC nanoparticles. As mentioned earlier, carbon nanostructures can also be grown at high substrate temperature [6-8]. The structuring evolution from amorphous carbon to nanoparticles growth by PLD has been studied in detail with respect to background gas and substrate temperature [7]. Transition of smooth amorphous films to nanostructuring occurred together with phase change from diamond-like/sp ${ }^{3}$ to graphitic/ $\mathrm{sp}^{2}$ phase, when substrate is heated, or with the presence of $\mathrm{He}$, or both [7]. It was noted that substrate heating or background gas would not promote nanostructures formation without sacrificing the $\mathrm{sp}^{3}$ phases in the films. In contrast, our results have shown that nanostructures with $\mathrm{sp}^{3}$ phases can be directly grown without substrate heating and in vacuum. The $\mathrm{sp}^{3}$ fraction of the nanostructured DLC film C0 was also higher than those deposited at high temperature, as can be been in its relatively lower $D$-peak as compared to those reported by others $[7,8]$. Thus, the morphological and the $\mathrm{sp}^{3} /$ diamond-like characteristics of the films should be explained taking into 


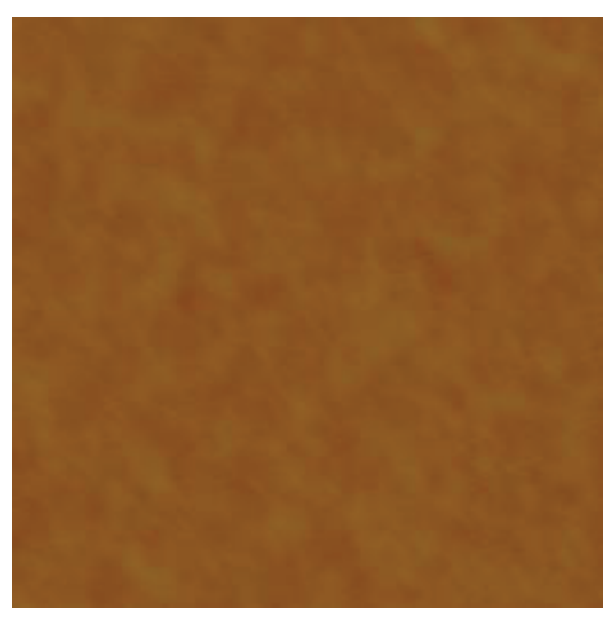

(a)

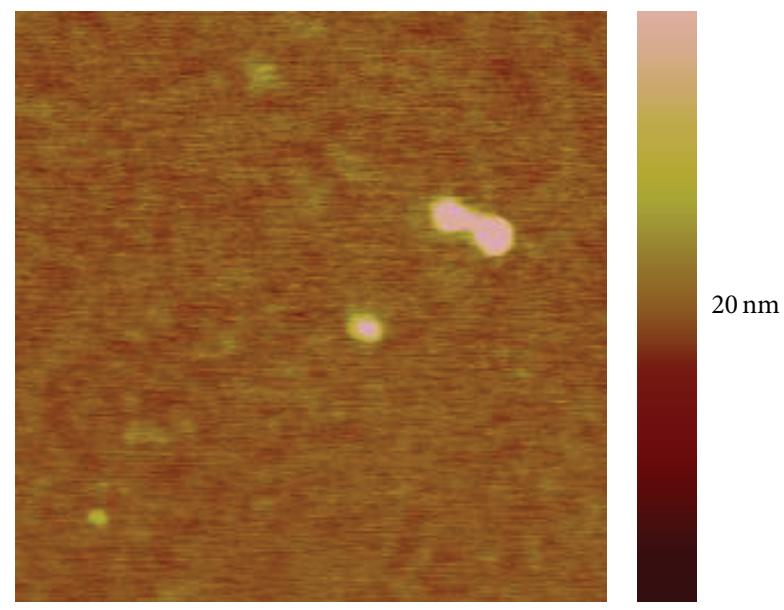

(b)

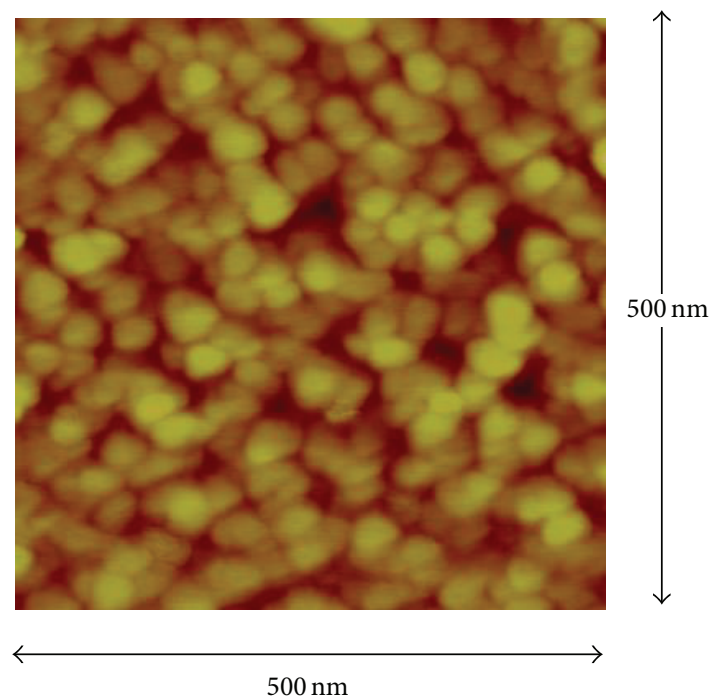

(c)

FIGURE 4: AFM images of deposited samples at (a) on-axis A90 and off-axis position, (b) C25, and (c) C0 (500 nm $\times 500 \mathrm{~nm}, 20 \mathrm{~nm}$ scale bar). 


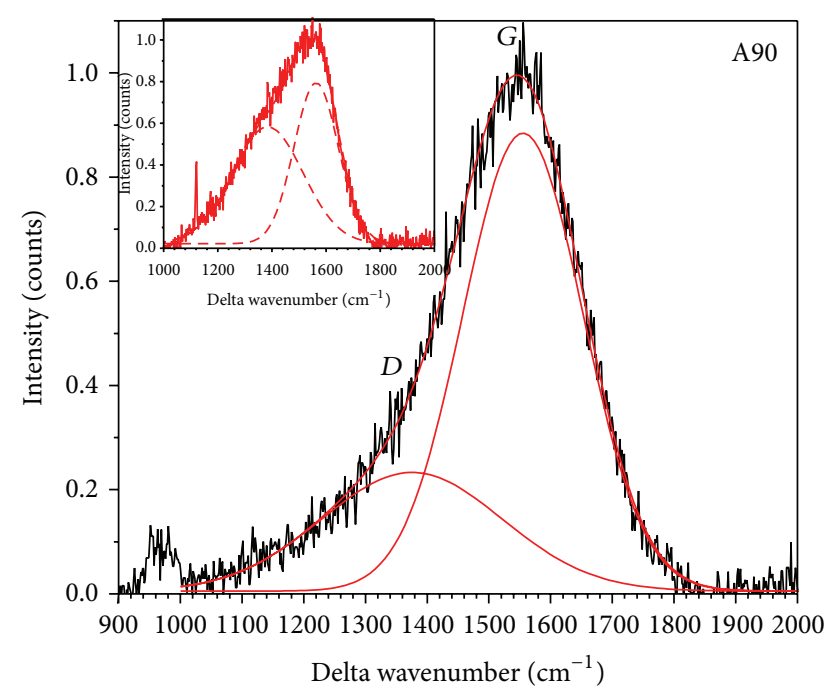

(a)

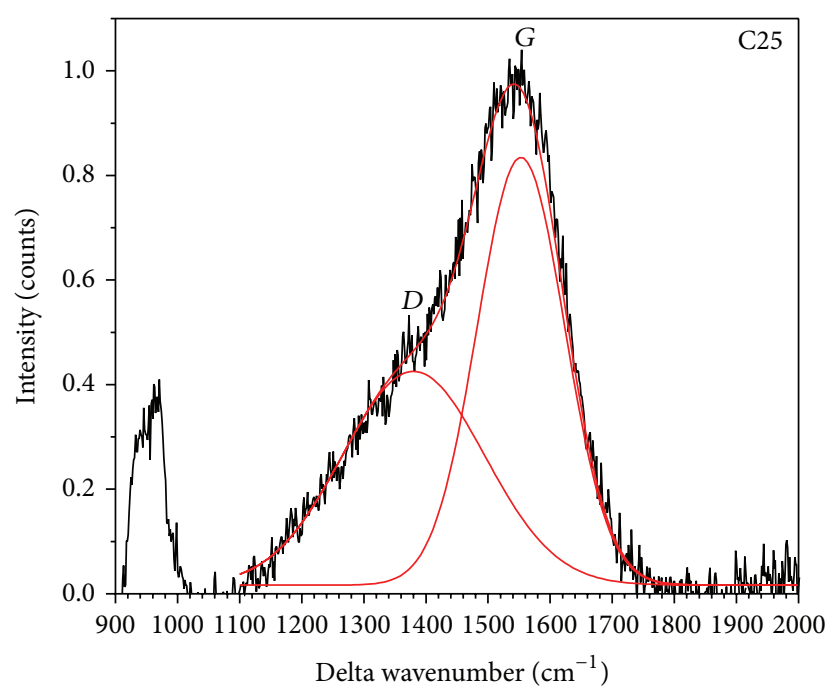

(b)

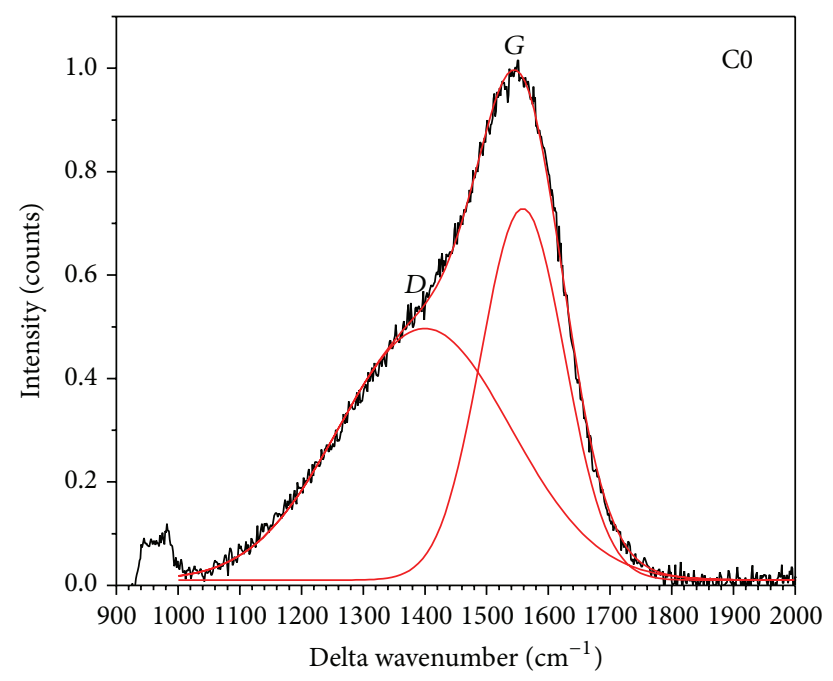

(c)

FIGURE 5: Raman spectra of deposited samples at (a) on-axis A90, with Raman spectra of a commercial DLC sample in the inset, (b) off-axis $\mathrm{C} 25$, and (c) off-axis position C0.

account the combined effects on the deposition configuration based on the following consideration.

Firstly, the structures obtained here are similar to those obtained by oblique angle deposition where columnar structure or even periodic arrays of nanostructure were produced $[18,19]$. In the reports, forward directed plume was utilized, but with the substrate positioned such that high density of deposition flux arrives at an angle with the substrate normal. Materials growth was explained by deposition of flux with arbitrarily chosen incidence angle, vapor condensation followed by shadowing, and surface diffusion processed that finally results in preferential structural growth. Micro- and nanostructures were also detected in our earlier work when the substrates were placed directly on the target surface where deposition relying mostly on backward scattered plume [20]. In the current configuration, forward directed plasma plume arrived at $\alpha=0^{\circ}$ effective angle (plasma plume is parallel to the substrate) onto the substrate almost immediately after plume generation and expansion for sample $\mathrm{C} 0$ as the substrate was positioned $5 \mathrm{~mm}$ from the target. The intense plasma density results in small and homogeneous nanostructures. Some sparse nanosized particles were found on sample C25 which was $\sim 3 \mathrm{~cm}$ from the target at larger effective angle.

Secondly, the formation of $\mathrm{sp}^{3}$ hybridized carbon has been ascribed mainly to subplantation process. This process of penetration of energetic species into subsurface layer can result in a lower surface resistivity because low $\mathrm{sp}^{3}$ layer will remain on the surface. The penetration also results in slower growth rate of DLC on carbide-forming Si because of the formation of an interface region, as observed in our previous work [21]. One of the crucial requirements for subplantation is that the ion energy has to be sufficiency high [22]. In the report, DLC films with $\mathrm{sp}^{3}$ fraction above $50 \%$ were obtained 
with ion energy of $\sim 30 \mathrm{eV}$. In this experiment, the ion energy was $\sim 35 \mathrm{eV}$ for sample $\mathrm{C} 0$ that suggests some amount of $\mathrm{sp}^{3}$ is still possible. The formation of larger clusters at small plume angle also suggests that other processes such as condensation or quenching of moderate energy $\mathrm{C}$ species into clusters coexisted. Thus, the nanostructured DLC films have poor adherence as compared to a solely subplanted DLC film. The condensed nanoclusters acted as seeds for nanostructures formation on the off-axis substrate.

\section{Conclusion}

Highly energetic ionized species were ejected perpendicular of the target that produced ultrasmooth DLC film in PLD. Less energetic ions travel at small angle from the target and result in nanostructured DLC films in vacuum and at room temperature. This was achieved in a simple deposition configuration when the substrate was placed at $45^{\circ}, 5 \mathrm{~mm}$ from the target where the plume density was sufficiently high. The formation of nanostructured DLC in this deposition configuration involved oblique angle deposition that gives rise to nanostructural growth, subplantation process by moderately energetic species, and quenching of $\mathrm{C}$ clusters in the plasma plume that subsequently seeded the nanostructural growth. The results also suggest that the method can be used to obtain nanostructured growth in other materials in PLD.

\section{Conflict of Interests}

The authors declare that there is no conflict of interests regarding the publication of this paper.

\section{Acknowledgments}

The authors acknowledge the support from Telekom Malaysia (RDTC/130823), University of Malaya (UM.C/625/1/HIR/ 231), and Ministry of Higher Education, Malaysia (FRGS/1/ 2013/SG06/MMU/03/2).

\section{References}

[1] D. L. Pappas, K. L. Saenger, J. Bruley et al., "Pulsed laser deposition of diamond-like carbon films," Journal of Applied Physics, vol. 71, no. 11, pp. 5675-5684, 1992.

[2] A. A. Voevodin and M. S. Donley, "Preparation of amorphous diamond-like carbon by pulsed laser deposition: a critical review," Surface and Coatings Technology, vol. 82, no. 3, pp. 199213, 1996.

[3] Y. Lifshitz, S. R. Kasi, J. W. Rabalais, and W. Eckstein, "Subplantation model for film growth from hyperthermal species," Physical Review B, vol. 41, no. 15, pp. 10468-10480, 1990.

[4] M. Moseler, P. Gumbsch, C. Casiraghi, A. C. Ferrari, and J. Robertson, "The ultrasmoothness of diamond-like carbon surfaces," Science, vol. 309, pp. 1545-1548, 2005.

[5] A. Erdemir and C. Donnet, "Tribology of diamond-like carbon films: recent progress and future prospects," Journal of Physics D: Applied Physics, vol. 39, no. 18, 2006.

[6] T. Ikuno, S. Honda, K. Aoki, K. Oura, and M. Katayama, "Carbon nanostructures grown on graphite substrates without catalyst by pulsed laser deposition," Japanese Journal of Applied Physics, vol. 45, pp. 2872-2874, 2006.

[7] E. Cappelli, S. Orlando, G. Mattei, C. Scilletta, F. Corticelli, and P. Ascarelli, "Nano-structured oriented carbon films grown by PLD and CVD methods," Applied Physics A: Materials Science and Processing, vol. 79, no. 8, pp. 2063-2068, 2004.

[8] X. D. Zhu, X. H. Wen, R. J. Zhan, H. Naramoto, and F. ArefiKhonsari, "Control of characteristic lengths for self-organized nanostructures of amorphous carbon," Journal of Physics: Condensed Matter, vol. 16, no. 8, pp. 1175-1181, 2004.

[9] D. B. Geohegan, A. A. Puretzky, G. Duscher, and S. J. Pennycook, "Time-resolved imaging of gas phase nanoparticle synthesis by laser ablation," Applied Physics Letters, vol. 72, no. 23, pp. 2987-2989, 1998.

[10] Y. Nakata, J. Muramoto, T. Okada, and M. Maeda, "Particle dynamics during nanoparticle synthesis by laser ablation in a background gas," Journal of Applied Physics, vol. 91, no. 3, pp. 1640-1643, 2002.

[11] P. M. Ossi, C. E. Bottani, and A. Miotello, "Pulsed-laser deposition of carbon: from DLC to cluster-assembled films," Thin Solid Films, vol. 482, no. 1-2, pp. 2-8, 2005.

[12] S. J. Henley, J. D. Carey, and S. R. P. Silva, "Room temperature photoluminescence from nanostructured amorphous carbon," Applied Physics Letters, vol. 85, no. 25, pp. 6236-6238, 2004.

[13] A. Zani, D. Dellasega, V. Russo, and M. Passoni, "Ultra-low density carbon foams produced by pulsed laser deposition," Carbon, vol. 56, pp. 358-365, 2013.

[14] D. Bolgiaghi, A. Miotello, P. Mosaner, P. M. Ossi, and G. Radnoczi, "Pulsed laser deposition of glass-like cluster assembled carbon films," Carbon, vol. 43, no. 10, pp. 2122-2127, 2005.

[15] A. C. Ferrari and J. Robertson, "Interpretation of Raman spectra of disordered and amorphous carbon," Physical Review BCondensed Matter and Materials Physics, vol. 61, no. 20, pp. 14095-14107, 2000.

[16] K. W. R. Gilkes, S. Prawer, K. W. Nugent et al., "Direct quantitative detection of the $\mathrm{sp}^{3}$ bonding in diamond-like carbon films using ultraviolet and visible Raman spectroscopy," Journal of Applied Physics, vol. 87, no. 10, pp. 7283-7289, 2000.

[17] T. Nakamura, Y. Mochidzuki, and S. Sato, "Synthesis of monodispersed DLC nanoparticles in intense optical field by femtosecond laser ablation of liquid benzene," in Proceedings of the Conference on Lasers Electro-Optics (CLEO'07), pp. 1-2, IEEE, Baltimore, Md, USA, May 2007.

[18] J. J. Steele and M. J. Brett, "Nanostructure engineering in porous columnar thin films: recent advances," Journal of Materials Science: Materials in Electronics, vol. 18, no. 4, pp. 367-379, 2007.

[19] D. Vick, Y. Y. Tsui, M. J. Brett, and R. Fedosejevs, "Production of porous carbon thin films by pulsed laser deposition," Thin Solid Films, vol. 350, no. 1, pp. 49-52, 1999.

[20] S. S. Yap and T. Y. Tou, "Target-plane deposition of diamondlike carbon in pulsed laser ablation of graphite," Applied Surface Science, vol. 253, no. 24, pp. 9521-9524, 2007.

[21] S. S. Yap, W. O. Siew, C. H. Nee, and T. Y. Tou, "Parametric studies of diamond-like carbon by pulsed Nd:YAG laser deposition," Diamond and Related Materials, vol. 20, no. 3, pp. 294-298, 2011.

[22] M. Chhowalla, J. Robertson, C. W. Chen et al., "Influence of ion energy and substrate temperature on the optical and electronic properties of tetrahedral amorphous carbon (ta-C) films," Journal of Applied Physics, vol. 81, no. 1, pp. 139-145, 1997. 

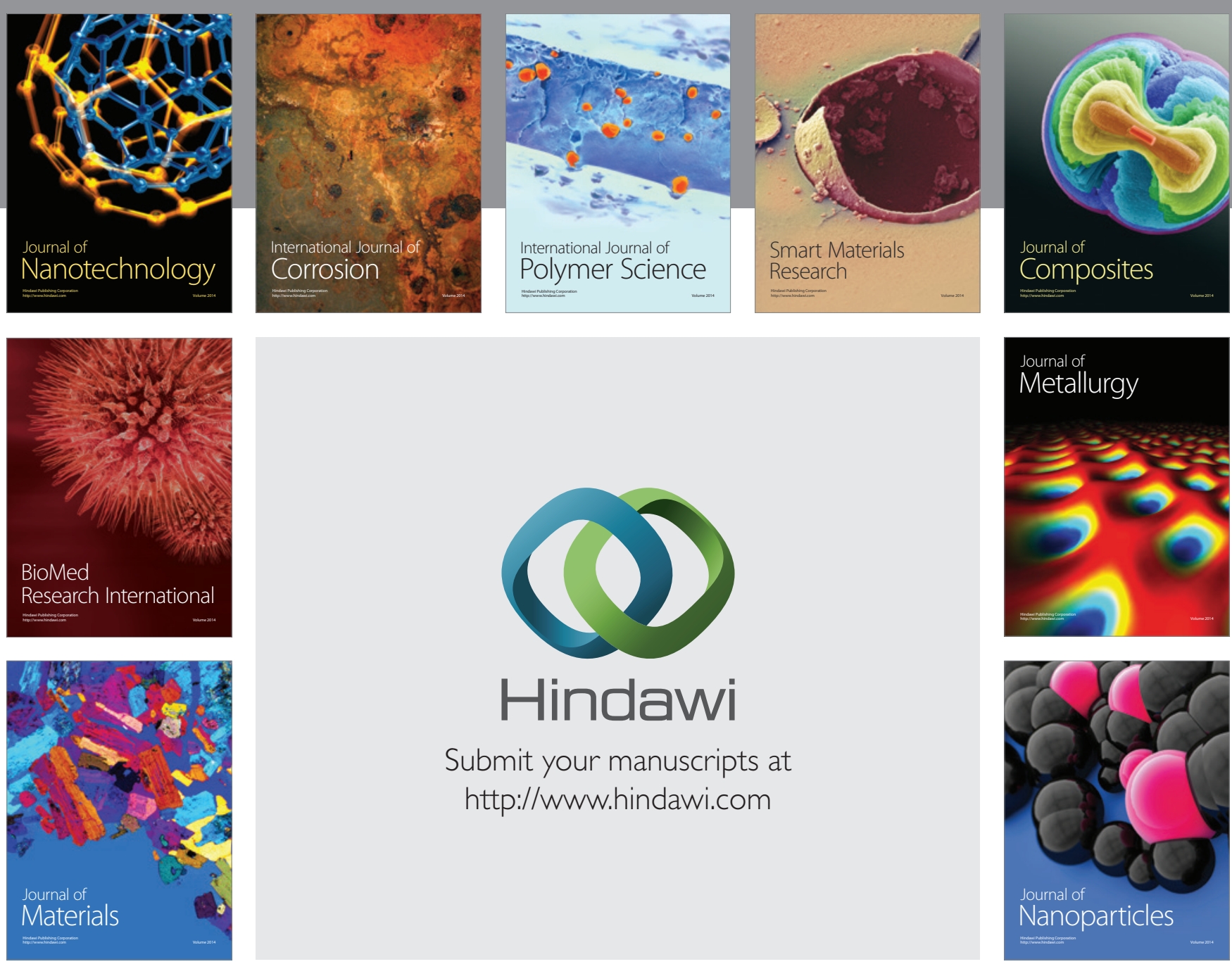

Submit your manuscripts at http://www.hindawi.com
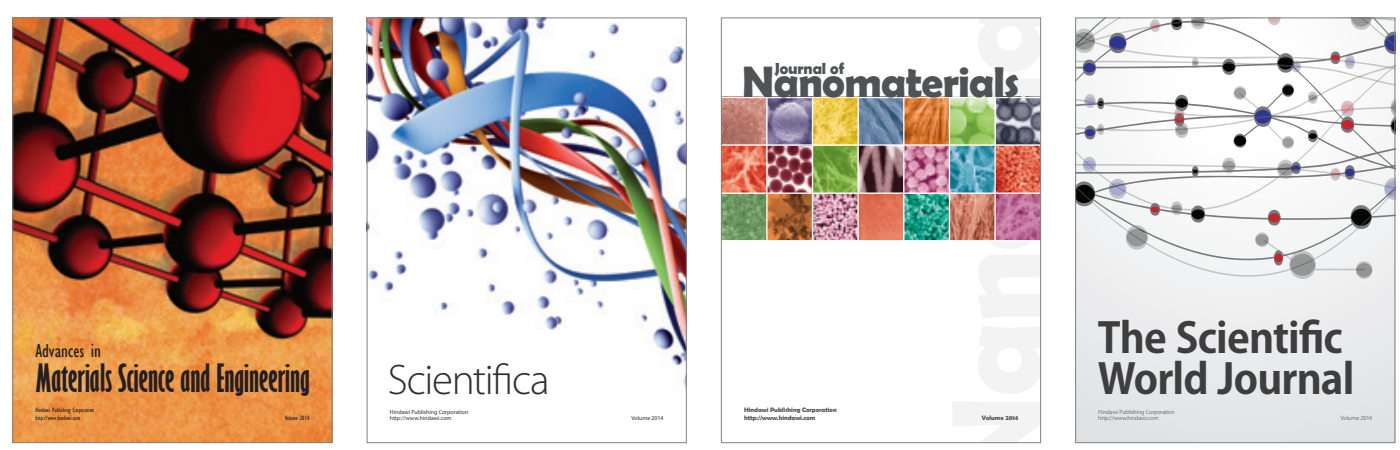

\section{The Scientific World Journal}
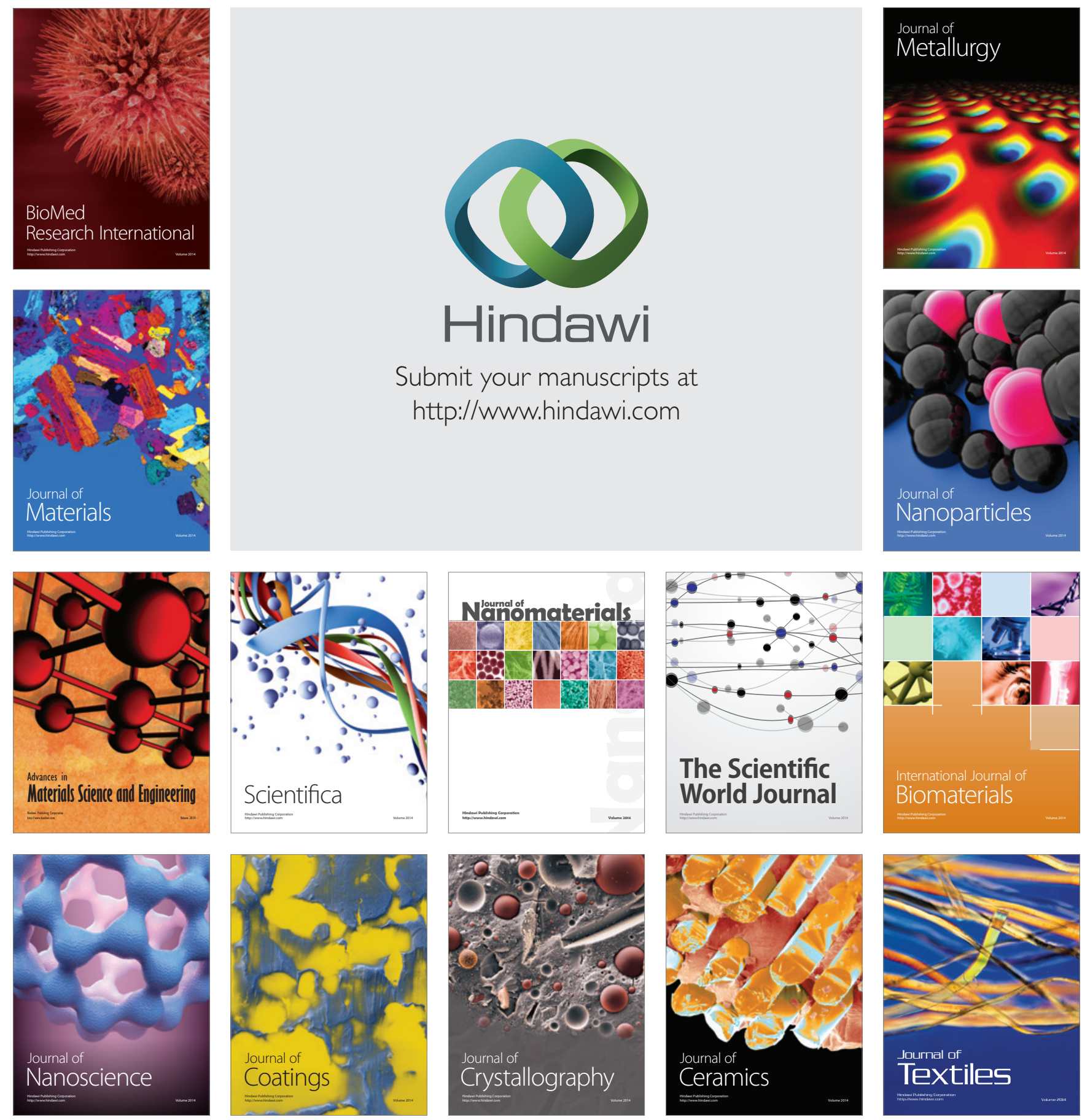